\title{
Simulation and Computational Study of Graphene Oxide Nanocarriers Loaded in the Absorption and Release of the Anticancer Drug of Camptothecin
}

\section{Omid Moradi}

Islamic Azad University

Leila Mahdavian ( $\triangle$ mahdavian_leila@yahoo.com )

Doroud branch, IAU https://orcid.org/0000-0001-9582-4667

\section{Research Article}

Keywords: Nano-graphene Oxide (NGO), Anti-cancer drug of camptothecin, Nano-drug carrier, Thermodynamic parameters, Totomersm process.

Posted Date: June 1st, 2021

DOI: https://doi.org/10.21203/rs.3.rs-501347/v1

License: (c) (i) This work is licensed under a Creative Commons Attribution 4.0 International License.

Read Full License

Version of Record: A version of this preprint was published at Journal of Molecular Modeling on August 17th, 2021. See the published version at https://doi.org/10.1007/s00894-021-04865-3. 


\title{
Simulation and computational study of graphene oxide nanocarriers loaded in the absorption and release of the anticancer drug of camptothecin
}

\author{
Omid Moradi ${ }^{1}$ and Leila Mahdavian ${ }^{2 *}$ \\ ${ }^{1}$ Department of Chemistry, Faculty of Science, Shahr-e-Qods Branch, Islamic Azad \\ University, Tehran, Iran \\ ${ }^{2}$ Department of Chemistry, Doroud Branch, Islamic Azad University, P.O. Box: 133. Doroud. \\ Iran. \\ Tel \& Fax: +986643232626 \\ *Corresponding Author: Mahdavian_leila@yahoo.com \\ Mahdavian@iau-doroud.ac.ir
}

\begin{abstract}
The structure of nano- graphene oxide, due to its special properties such as hydrophilicity, special surface and suitable biocompatibility, the possibility of high loading of hydrophilic and hydrophobic drugs, has attracted special attention in drug release. In this study, after simulating and optimizing the structure of nano-graphene and then nano-graphene oxide (NGO), it was used to load the anti-cancer drug of camptothecin (CA) in aqueous medium and the optimal conditions for achieving maximum loading efficiency of the drug were investigated. Due to the structure of the drug, there are two forms of lactone ring and carboxylate, which if the lactone ring form is predominant, the effectiveness of the drug is increased, which depends on the $\mathrm{pH}$ of environment.

The calculated thermodynamic and structural results show that the solubility of the drug in relation to nano-graphene and its lactone ring state are maintained by using nano-graphene oxide. By folic acid as an intermediate in aqueous medium, the drug is released to form of lactone ring and is increased the effectiveness of drug. Lactony is maintained the drug structure and is increased the drug effectiveness. The results show that the presence of the ring in the drug structure and its binding to the mediator of folic acid to nano-graphene oxide is a stabilizing factor of keto tautomer. The calculation of vibrational frequencies show that the presence of folic acid intermediate reduces the vibrational frequency of the hydroxyl group $(\mathrm{OH})$ so that its absorption energy $\left(E_{a d}\right)$ is equal to the lowest value 65.24 a.u.
\end{abstract}

Keywords: Nano-graphene Oxide (NGO), Anti-cancer drug of camptothecin, Nanodrug carrier, Thermodynamic parameters, Totomersm process. 


\section{Introduction}

It would be great if medications were administered in a way that met exactly the physiological needs at the right time or in the right place. The purpose of designing drug delivery systems based on nano-composites is to overcome the defects and defects of conventional drug formulations, reduce the frequency and frequency of drug use or increase the effect of the drug by focusing on the desired location, reduce the amount of drug required and provide uniform drug delivery [1-3].

One of the drug carriers that have received special attention in the last decade is graphene and graphene derivatives, which have provided a diverse context for achieving the goals of control-oriented treatment [4]. Features and properties of this nano-carrier, including; Low toxicity, unique shape and geometry, high ability to load biomaterials, easy synthesis and low cost, have led to increased attention to their use in the delivery of drugs, proteins and genes. The efficiency of graphene nanoparticles and their derivatives, such as graphene oxide, is increased in the drug delivery system by surface modification by polymers and other nanoparticles. Properties that have received the most attention in the field of drug delivery include; Unique surface chemistry, high purity and biocompatibility and low toxicity. At the same level as other nano-carriers, graphene is four times more capable of transporting and transporting drugs (approximately 2,600 g. $\mathrm{m}^{-2}$ ) [5]. Another important characteristic of nano-graphene and nano- graphene oxide in the field of drug delivery is the loading ratio (weight ratio of loaded drug to carrier), which in the case of graphene nanomaterials can be up to $200 \%$, which is significantly higher than other nano-materials and other drug delivery systems [6].

It is noteworthy that if the structure of nano-graphene and nano-graphene oxide becomes too hard, it will damage the cells. Therefore, their disadvantages can be eliminated by modifying the surface of these nanostructures. Due to the surface chemistry, nano-graphene is highly hydrophobic and does not disperse well in water and requires surfactants or surface modifiers for biological applications. In contrast, nano-graphene oxide is hydrophilic and can be dispersed in water to form a stable colloid (nano-graphene has a non-covalent bond but nano-graphene oxide has a covalent bond). Nano-graphene oxide with its epoxy, hydroxyl and acidic hydrophilic groups has greatly increased its biocompatibility [7]. Although nano-graphene oxide 
is soluble in water, it accumulates in physiological buffers in the presence of salt due to screening. Improving the surface and increasing the efficiency of nano-graphene and nano-graphene oxide, in general, increasing the solubility and dissolution of drugcarrying nano-materials in the drug delivery system, improves the performance and reduces the toxicity of these materials. For this purpose are used different materials and polymers, the role they play in the drug delivery system is divided into three categories [8]: i- as a carrier, ii- as a biologically active component in the delivery of a drug, protein or peptide, and iii- as a spacer molecule.

One of the drugs proposed for the treatment of cancer is camptothecin, a five-ring alkaloid extracted from the Chinese tree Camptotheca acuminata, which was isolated by Wall et al. [9]. It is a potent anti-cancer drug with a wide range of anti-tumor effects in animal models of lung, prostate, breast, colon, stomach, bladder, ovarian and melanoma cancers and exerts its effect by inhibiting the enzyme topoisomerase 1 . Structure of camptothecin is limited; A) Carboxylate form, b) Lactone form The therapeutic use of this drug, due to its instability at neutral $\mathrm{pH}$, low solubility in aqueous media $\left(8.3 \mathrm{mg} \cdot \mathrm{ml}^{-1}\right)$ and its unwanted toxicity effects. Figure 1 shows its optimized geometric structure. Depending on the $\mathrm{pH}$, there are two forms of camptothecin; Lactone and carboxylate form. At $\mathrm{pH}<5$, the drug is in the lactone form, which is the active form of the drug, and its antitumor effects depend on the presence of a lactone ring.

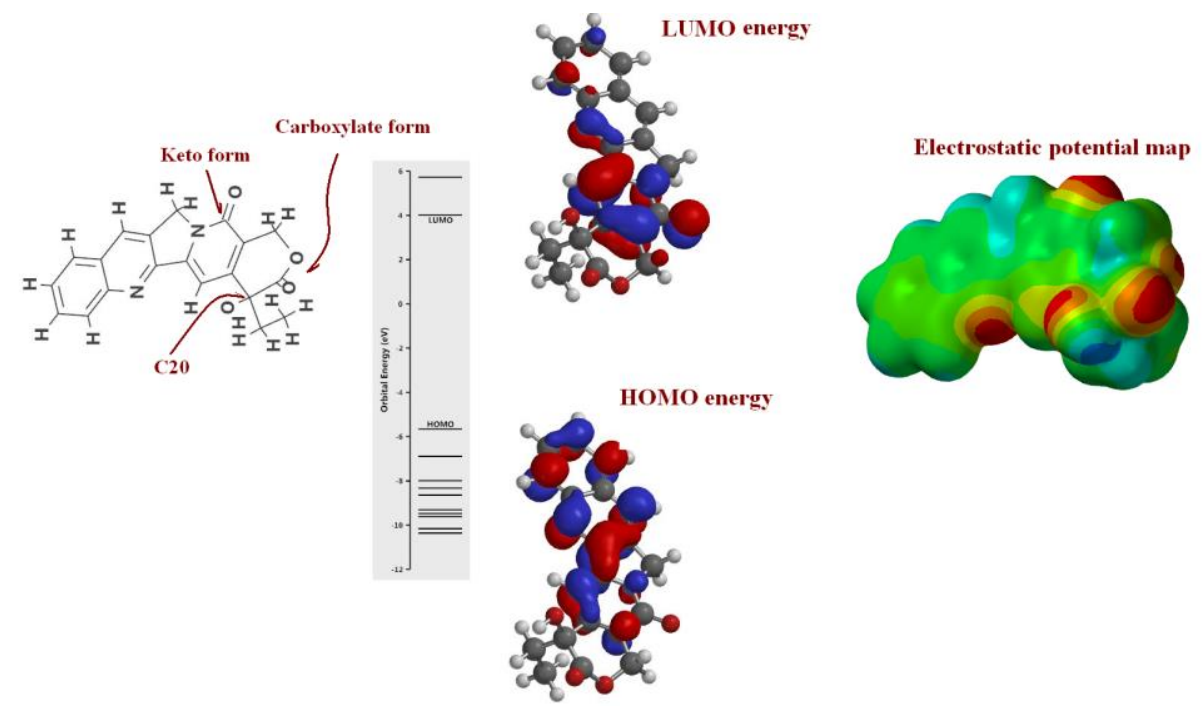

Figure 1. Geometric and optimized structure of the anticancer drug of camptothecin and its lactone and carboxylate forms. 
In this study, using nano-graphene oxide (NGO) modified by folic acid, are investigated the release rate of the drug and its effective structure because the best form of effectiveness of camptothecin is its lactone form, whose is examined structural changes in the presence of nano-graphene oxide as the drug carrier. Instead of spreading rapidly, these nanoparticles slowly decompose and release the drug. In Figure 2, are optimized the structures of nano-graphene, nano-graphene oxide and nano-graphene oxide/folic acid and is simulated their interaction with the anticancer drug of camptothecin. Then, thermodynamic and electrical parameters and their compatibility with the environment have been studied and evaluated by DFT computational method.
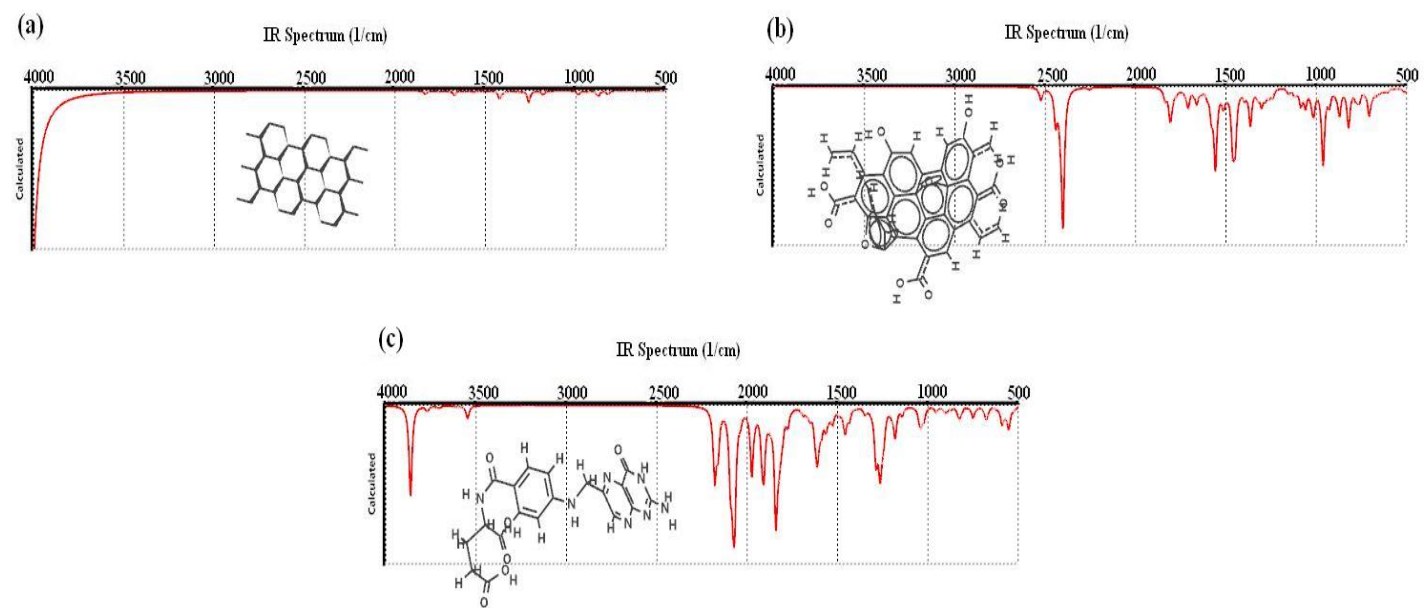

Figure 2. IR spectrum with optimized structure a) graphene, b) graphene oxide and c) folic acid.

\section{Calculation methods}

All calculations are performed with the Wavefunction Spartan series of programs [10]. Geometry optimization is performed using scattering including density functional theory with M06-2X method and 6-31G** base set in aqueous medium. This hybrid meta-exchange correlation function is used to model non-covalent interactions, especially those related to graphene-based systems [11]. The 6-31 G** base set is suitable for general computations on medium to large molecules, and research shows that M06-2X/6-31G** is an optimal level of theory for providing reliable and accurate results at the lowest cost for a variety of systems [12 -14]. The structure of nano-graphene, nano-graphene oxide, nano-graphene oxide/folic acid has been optimized with the anticancer drug of camptothecin and the interaction between them in the presence of solvent, the optimal structure of which is shown in Figures 1 
and 2. The energy associated with Ead absorption is calculated by the interaction between them using the following equation:

$\mathrm{E}_{\mathrm{ad}}=\mathrm{E}_{\mathrm{NS}}-\mathrm{D}+\left[\mathrm{E}_{\mathrm{D}}+\mathrm{E}_{\mathrm{NS}}\right]$

ENS-D, ED and ENS are the total energy of the complex created in the absorption of the drug on the nano-surface, the total energy of the drug and the nano-surface alone or with intermediate, respectively. To investigation of the electrical properties and structural of compounds and intermediates formed between them, is calculated the energy levels of Highest occupied molecule orbital (HOMO) and Lowest un-occupied molecular orbital (LUMO) by DFT method [15]. The calculation of HOMO and LUMO energies in molecules is often done with the aim of predicting their activity characteristics, nano-surface behavior, and electron conduction. The energy difference between the two surfaces is called the energy gap $\left(\mathrm{E}_{\mathrm{g}}\right)$, which indicates the electron transfer of the structure of the molecule. In density function theory (DFT), any chemical system is defined by an electronic chemical potential $(\eta)$ and an absolute chemical hardness $(\mu)$. These parameters are the activity descriptors of chemical species that can be used to represent the electron transfer process in hybrid systems. Chemical potential indicates the tendency of a super-electron to escape equilibrium and is defined as a derivative of ground-state electronic energy (E) with respect to the number of electrons $(\mathrm{N})$. Absolute Chemical Hardness is the resistance to charge transfer. According to the energy level of LUMO and HOMO can be achieved structural parameters. Electron afficiency energy of the molecule is the difference between the base level with LUMO level energy and the ionization energy of the molecule is the difference between the base energy level with HOMO level energy. The total energy of these two surfaces is twice the electronegativity ( $\eta$ ) of the molecule, which is a measure of the hardness and softness of the molecule structure. Therefore, chemical or electronegative potential $(\eta)$, chemical hardness $(\mu)$, chemical softness $(\sigma)$, electrophile $(\omega)$, total softness $(\mathrm{S})$ and energy $\left(\Delta \mathrm{E}_{\text {back-dontion }}\right)$ using boundary molecular circuits are calculated by the following equations:

$\mathrm{E}_{\mathrm{g}}=\mathrm{E}_{\mathrm{LUMO}}-\mathrm{E}_{\mathrm{HOMO}}$

$\eta=-\left(\mathrm{E}_{\text {LUMO }}+\mathrm{E}_{\text {Hомо }}\right) / 2$

$\mu=\left(\mathrm{E}_{\mathrm{LUMO}}-\mathrm{E}_{\text {Hомо }}\right) / 2$

$\sigma=1-\mu$

$\omega=\eta^{2} /(2 \mu)$ 
$\Delta \mathrm{E}_{\text {back-donation }}=-\mu / 4$

Also, $\Delta \mathrm{N}$ represents the fraction of electrons transferred from the drug (D) to the nano-surface (NS) obtained from the following equation [16]:

$\Delta \mathrm{N}=\left(\eta_{N S}-\eta \mathrm{D}\right) / 2(\mu \mathrm{NS}-\mu \mathrm{D})$

As can be seen in Figure 1, there are two forms of ketone and carboxylate in the structure of camptothecin, the interaction of which with the nano-surface of nanographene oxide is investigated. The lactone form in the interaction can be in the form of the enol and ketone form, which are in totomeric interaction with each other.

\section{Results and discussion}

According to the presented materials, nano-graphene and its derivatives have defects, such as low solubility, which can be eliminated by modifying the surface of these nanostructures. Of course, this is especially true for nano-graphene, which is highly hydrophobic and lacks oxygen-containing hydrophilic groups, which play an important role in the solubility of aqueous solutions.

As shown in Figure 2, nano-graphene oxide is a highly oxidized form of chemically modified nano-graphene, which is decorated on the edges with oxygen-containing functional groups such as hydroxyl and epoxy on the base plate with carbonyl and carboxyl. These hydrophobic groups enable NGOs to act like an amphiphile and improve the water dispersion of NGOs [17-20]. Table 1 shows the thermodynamic and structural parameters of nano-surface and their modification by folic acid with the camptothecin anticancer drug (CA). The polar moment of nano-graphene is $0 \mathrm{D}$ while the dipole moment of NGO is equal to11.53 D, so it can have better biocompatibility than nano-graphene. These groups mostly provide a reaction site for hydrogen bonding between the drug and the nanotube. The symmetric group of nano-graphene $\mathrm{C}_{2 \mathrm{~h}}$ means that it has a $\delta_{\mathrm{h}}$ plane that cannot be optically active, while nano-graphene oxide has a symmetric group $\mathrm{C}_{1}$ and is optically active. Graphene oxide has been extensively studied in many areas, especially in biological applications and drug delivery, due to its unique physicochemical properties such as excellent solubility, large specific surface area and low toxicity [21, 22]. Facilitate on its surface and edge through mechanisms such as $\pi-\pi$ accumulation, electrostatic interactions and hydrogen bonding. Therefore, drug loading capacity from NGOs is typically higher than other nanoparticle-based carriers. 
To make drug carriers with good compatibility and control the behavior of these nano-materials in the biological system, changes must be made in their surface chemistry. There are two main methods for changing the surface of nano-graphene and nano-graphene oxide: 1- non-covalent modification method and 2- covalent modification method.

The non-covalent method is the connection by van der Waals and electrostatic forces without any change in the natural structure of the surface. Graphene oxide modified by this method has been used in the fields of surfactant adsorption, interaction with porphyrins and biological molecules such as DNA and peptides [23]. Modifications with this method include van der Waals forces, electrostatic interaction, hydrogen bonding, and foot-to-foot bonding to correct nano-graphene and nano-graphene oxide. The method of non-covalent modification with organic polymers and molecules is foot-to-foot bonding, for example beta-cyclodextrin to nano-graphene oxide by $\Pi-\Pi$ bonding [24]. Nano-graphene modified with folic acid by non-covalent modification method has been used for the delivery and delivery system of adriamycin [25]. Nanoparticles such as gold, silver, palladium, nickel, platinum and copper, $\mathrm{TiO}_{2}$, $\mathrm{ZnO}, \mathrm{MnO}_{2}, \mathrm{Co}_{3} \mathrm{O}_{4}$ and $\mathrm{Fe}_{3} \mathrm{O}_{4}$ can be used to modify nano-graphene and nanographene oxide by conjugation by foot for drug and other magnetic applications [26]. The method of covalent modification, such as atom doping or reacting with oxygen groups, is the nano-graphene oxide surface. Surface modification is easy with this method and the nanostructure modified with this method is mainly used in drug delivery. The presence of reactive oxygen groups on the surface of nano-graphene oxide sheets makes this nanostructure as a polymer base in reactions. Covalent changes are made in several ways, including: nucleophilic substitution, which is the main method and is done by attacking only alcohol, epoxy and acidic nano-graphene oxide groups by amino, hydroxyl and electron pair groups [27]. Increased electrophilicity and increased density. It is therefore a promising way to bind a variety of aromatic and aliphatic amino acids, amino acids and biomolecules, silanes, enzymes and polymers.

In Table 1, zero-point energy (ZPE) is the lowest possible energy that can have a quantum mechanical system, which is higher for nano-graphene oxide than for graphene. $\mathrm{H}^{\circ}$ (heat of reaction), $\mathrm{G}^{\mathrm{o}}$ (the free energy of Gibbs), $\mathrm{Cv}$ (heat capacity) and $S^{o}$ (enthalpy) are calculated and represented separately in aqueous solution. 
Comparing the two levels of nano-graphene and nano-graphene oxide, the thermodynamic values of nano-graphene oxide indicate more chemical activity of this surface. By comparing the structural properties of these two surfaces, nanographene oxide has a more electronegativity structure $(\eta=1.05 \mathrm{eV})$ and less chemical hardness and total chemical softness, and has more $\Delta$ Eback-dontion energy than nano-graphene oxide.

In the study, nano-graphene and nano-graphene oxide are modified by folic acid, which according to the structure, the type of interaction of graphene is considered as non-covalent bond and nano-graphene oxide is considered as covalent bond.

The computational data of Table 1 show that tendency of nano-graphene oxide to interact with folic acid is higher than nano-graphene and the dipole moment for nanographene is $1.08 \mathrm{D}$ while this polarity for nano-graphen oxide is equal to $18.41 \mathrm{D}$. Despite the binding of folic acid, has not been reduced the hydrophobicity of nanographene. Investigation of properties and structure electrical, gap energy $\left(\mathrm{E}_{\mathrm{g}}\right)$ and other calculated parameters; it is found that the nano-graphene oxide/folic acid composite has more structural stability than the nano-graphene/folic acid.

Table 1: Thermodynamic and structural properties of nano-graphene (NG), nano-graphene oxide (NGO), folic acid (FA), nano-graphene/folic acid (NG/FA) composite, nano-graphene oxide/folic acid composite (NGO/FA) and the camptothecin anti-cancer drug (CA).

\begin{tabular}{|c|c|c|c|c|c|c|c|c|c|}
\hline \multicolumn{10}{|c|}{ Thermodynamic properties } \\
\hline & $\begin{array}{l}\mathbf{E}_{\text {total }} \\
\text { a.u. }\end{array}$ & $\begin{array}{c}\text { Dipole } \\
\text { mome } \\
\text { nt } \\
\text { /D } \\
\end{array}$ & $\begin{array}{c}\text { Symmetry } \\
\text { group }\end{array}$ & $\begin{array}{c}\text { Zero point } \\
\text { energy } \\
(\mathrm{ZPE}) / \mathbf{k J}^{-} \mathrm{mol}^{-1}\end{array}$ & \multicolumn{2}{|c|}{$\mathrm{H}^{\mathrm{o}} / \mathbf{a} . \mathbf{u}$. } & $\begin{array}{l}\mathbf{G}^{\mathbf{0}} \\
\text { a.u. }\end{array}$ & 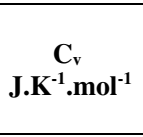 & $\begin{array}{c}\mathbf{S}^{\mathbf{o}} \\
\mathbf{J} \cdot \mathbf{K}^{-1} \cdot \mathbf{m o l}^{-1}\end{array}$ \\
\hline NG & -1344.20 & 0.00 & $\mathrm{C}_{2 \mathrm{~h}}$ & 519.58 & \multicolumn{2}{|c|}{-1343.98} & -1344.05 & 287.19 & 602.64 \\
\hline NGO & -2195.88 & 11.53 & $\mathrm{C} 1$ & 861.08 & \multicolumn{2}{|c|}{-2195.51} & -2195.61 & 432.77 & 838.21 \\
\hline $\begin{array}{l}\text { Folic } \\
\text { Acid } \\
\end{array}$ & -1534.77 & 6.97 & $\mathrm{C} 1$ & 1235.88 & \multicolumn{2}{|c|}{-1534.28} & -1534.35 & 305.54 & 667.46 \\
\hline NG/FA & -1789.21 & 1.08 & $\mathrm{C} 1$ & 1489.23 & \multicolumn{2}{|c|}{-1805.69} & -1805.91 & 512.32 & 967.86 \\
\hline NGO/FA & -3661.72 & 18.41 & $\mathrm{C} 1$ & 1966.62 & \multicolumn{2}{|c|}{-3660.91} & -3661.05 & 716.92 & 1202.80 \\
\hline CA & -1160.34 & 5.35 & $\mathrm{C} 1$ & 997.72 & \multicolumn{2}{|c|}{-1159.84} & -1159.90 & 227.70 & 532.41 \\
\hline \multicolumn{10}{|c|}{ DOS calculations } \\
\hline & $\begin{array}{c}\text { ELumo }_{\text {LeV }} \\
\text { /eV }\end{array}$ & $\begin{array}{c}\text { Eномо }_{\text {ном }} \\
/ \mathrm{eV}\end{array}$ & $\mathbf{E}_{\mathrm{g}} / \mathrm{eV}$ & $\eta / e V$ & $\mu / \mathrm{eV}$ & $\sigma / \mathbf{e V}$ & $\omega / \mathbf{e V}$ & S/eV & $\begin{array}{l}\Delta \mathbf{E}_{\mathbf{b}-\mathbf{d}} \\
/ \mathrm{eV}\end{array}$ \\
\hline NG & 2.00 & -2.54 & 4.54 & 0.27 & 2.27 & -1.27 & 0.016 & 0.22 & -0.57 \\
\hline NGO & -0.27 & -1.83 & 1.56 & 1.05 & 0.78 & 0.22 & 0.71 & 0.64 & -0.20 \\
\hline $\begin{array}{l}\text { Folic } \\
\text { Acid }\end{array}$ & 5.46 & -1.93 & 7.39 & -1.76 & 3.69 & -2.69 & 0.42 & 0.14 & -0.92 \\
\hline NG/FA & 2.14 & -1.98 & 4.12 & -0.08 & 2.06 & -1.06 & 0.0016 & 0.24 & -0.52 \\
\hline NGO/FA & 0.37 & -3.23 & 3.6 & 1.43 & 1.8 & -0.8 & 0.57 & 0.28 & -0.45 \\
\hline CA & 4.01 & -5.66 & 9.67 & 0.83 & 4.84 & -3.84 & 0.07 & 0.103 & -1.21 \\
\hline
\end{tabular}


Therefore, the binding of the anticancer drug of camptothecin can be investigated as direct binding to nano-graphene oxide (in the form of enol and keto) and also binding to folic acid intermediate (in the form of enol and keto). Figure 3 shows the drug approaching nano-graphene oxide. The $\mathrm{OH}$ of carbon 20 (C20) approaches the functional group of nano-graphene oxide. It is attached to the surface by a covalent bond when it loses a molecule of water. In this connection, the two states of keto and enol are connected as totomerists with nano-surface. Table 2 shows the computational data of the direct connection (without intermediaries) to the drug.

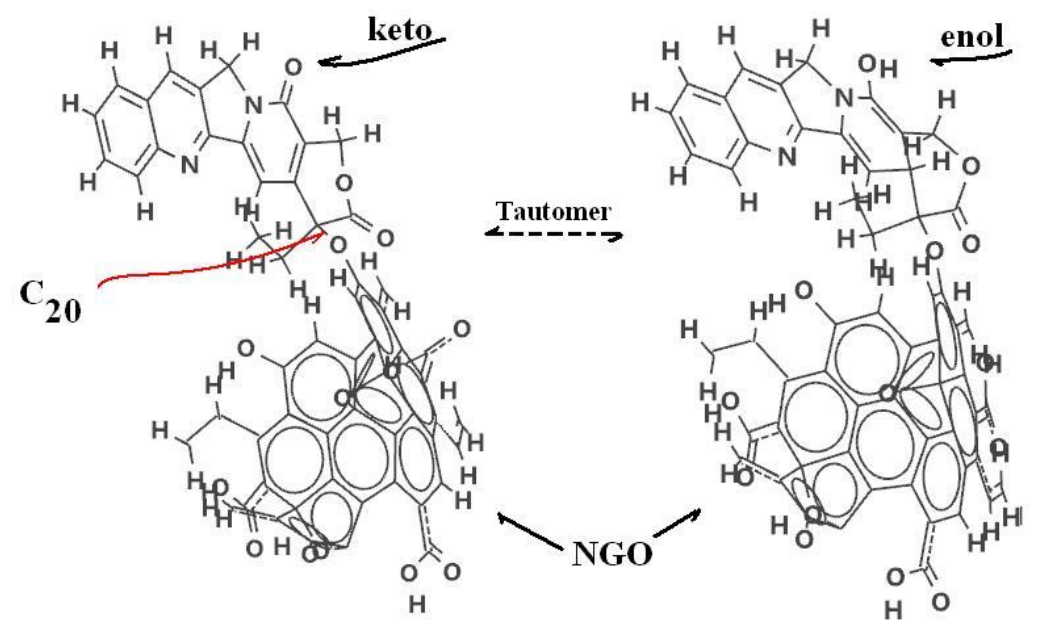

Figure 3. Optimized structure of binding of the anticancer drug of camptothecin to nano-graphene oxide as ketone form totomerism.

The effective form of the camptothecin anti-cancer drug is its lactone form. Using nano-graphene oxide and the involvement of the carboxyl functional group, the lactone form of the drug is active, which due to the totomerism process can be in the form of enol and keto. Table 2 shows the thermodynamic and structural parameters of the direct binding of the drug to nano-graphene oxide. The dipole moment of the keto form is $19.20 \mathrm{D}$, which is less than its enol form, which indicates that the keto form of the drug in this junction shows less structural changes. The calculated thermodynamic values show that the keto form is more likely in this composite that the heat of reaction formation in the keto form is equal to -3290.04 a.u. and the enol form equal to -3277.39 a.u., the Gibbs free energy and entropy also indicate a higher probability of the keto form in the direct binding of the drug to the nano-graphene oxide.

The gap energy $\left(E_{g}\right)$ for enol form is more than keto form, so the softness of the structure in this case is more than keto form, which can reduce the effectiveness of the drug. $\Delta \mathrm{N}$ is negative for the keto form, which indicates the transfer of electrons from 
the nano-surface to the drug, and positive for the enol form, which indicates the opposite.

Table 2. Thermodynamic and structural parameters of the camptothecin anticancer drug/nano-graphene oxide as keto form in totomerism.

\begin{tabular}{|c|c|c|c|c|c|c|c|c|c|c|c|}
\hline \multicolumn{12}{|c|}{ Thermodynamic properties } \\
\hline & $\begin{array}{l}\mathbf{E}_{\text {total }} \\
\text { a.u. }\end{array}$ & \multicolumn{2}{|c|}{$\begin{array}{c}\text { Dipole moment } \\
\text { /D }\end{array}$} & \multicolumn{2}{|c|}{$\begin{array}{c}\text { Zero point } \\
\text { energy } \\
\text { (ZPE)/kJ.mol }{ }^{-1}\end{array}$} & $\mathbf{H}^{\circ} /$ a.u. & \multicolumn{2}{|r|}{$\begin{array}{l}\mathbf{G}^{\mathbf{0}} \\
\text { a.u. }\end{array}$} & $\begin{array}{c}\mathbf{C}_{\mathbf{v}} \\
\mathbf{J} \cdot \mathbf{K}^{-1} \cdot \mathrm{mol}^{-1}\end{array}$ & \multicolumn{2}{|c|}{$\begin{array}{c}\mathrm{S}^{\mathbf{o}} \\
\mathbf{J} \cdot \mathbf{K}^{-1} \cdot \mathbf{m o l}^{-1}\end{array}$} \\
\hline Keto form & -3290.98 & \multicolumn{2}{|l|}{19.20} & \multicolumn{2}{|c|}{2350.78} & -3290.04 & \multicolumn{2}{|r|}{-3290.16} & 632.32 & \multicolumn{2}{|c|}{1003.82} \\
\hline Enol form & -3278.60 & \multicolumn{2}{|l|}{34.00} & \multicolumn{2}{|c|}{3064.92} & -3277.3 & \multicolumn{2}{|r|}{-3277.51} & 649.28 & \multicolumn{2}{|c|}{1059.29} \\
\hline \multicolumn{12}{|c|}{ DOS calculations } \\
\hline & $\begin{array}{c}\text { ELUMo }_{\text {LUV }} \\
/ \mathrm{eV}\end{array}$ & $\begin{array}{c}\text { Eномо }_{\text {ном }} \\
/ \mathrm{eV}\end{array}$ & $\mathbf{E}_{\mathrm{g}} /$ & & $\eta / e V$ & $\mu / \mathrm{eV}$ & $\sigma / \mathrm{eV}$ & $\omega / \mathbf{e}$ & S/eV & $\begin{array}{l}\Delta \mathbf{E}_{\text {b-d }} \\
/ \mathbf{e V}\end{array}$ & $\Delta \mathbf{N}$ \\
\hline Keto form & 5.43 & 0.78 & 4. & & -3.11 & 2.33 & -1.32 & 2.0 & 0.22 & -0.58 & -1.34 \\
\hline Enol form & 5.62 & 3.21 & 2. & & -4.42 & 1.21 & -0.21 & 8.0 & 0.41 & -0.30 & 6.36 \\
\hline
\end{tabular}

Alternatively, folic acid intermediate can be used to transfer and release the camptothecin anticancer drug. Folic acid is placed between nano-graphene oxide and the drug. Figure 4, the geometric structure of the two forms keto and enol of the drug is seen against nano-graphene oxide/folic acid. Folic acid is used to reduce blood complications and increase the effectiveness of some anticancer drugs. Table 3 shows the thermodynamic and structural data of the drug/folic acid/nano-graphene oxide complex in enol and keto form.

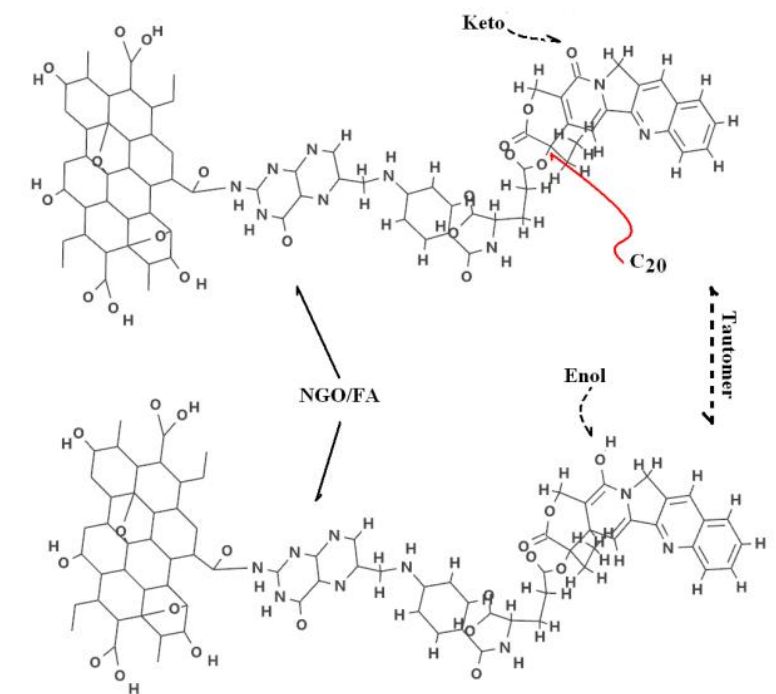

Figure 4. Optimized structure of the camptothecin anticancer drug with folic acid/nano-graphene oxide nano-composite in totomerism of enol $\leftrightarrow$ ketol.

The calculated dipole moment for both forms does not show a significant difference, but it is slightly higher for the enol form than for the ketone form. Comparing the thermodynamic parameters, the keto form of the drug/folic acid/graphene oxide nanocomposite is more likely to be very small; the difference in thermodynamic 
parameters between the two forms is very small. Thermodynamic data show that by the interaction of folic acid with nano-graphene oxide, a structural equilibrium occurs between the keto and anol forms.

Table 3. Thermodynamic and structural parameters of the camptothecin anticancer drug with folic acid/nano-graphene oxide nano-composite in totomerism of enol $\leftrightarrow \mathrm{ketol}$.

\begin{tabular}{|c|c|c|c|c|c|c|c|c|c|c|c|}
\hline \multicolumn{12}{|c|}{ Thermodynamic properties } \\
\hline & $\begin{array}{l}\text { Etotal }_{\text {a. }} \\
\text { a.u. }\end{array}$ & \multicolumn{2}{|c|}{$\begin{array}{c}\text { Dipole moment } \\
\text { /D }\end{array}$} & \multicolumn{2}{|c|}{$\begin{array}{c}\text { Zero point } \\
\text { energy } \\
\text { (ZPE)/kJ.mol }{ }^{-1}\end{array}$} & \multicolumn{2}{|c|}{$\mathbf{H}^{\circ} \%$ a.u. } & $\begin{array}{l}\mathbf{G}^{\mathbf{0}} \\
\text { a.u. }\end{array}$ & \multicolumn{2}{|c|}{$\begin{array}{c}\mathbf{C}_{\mathrm{v}} \\
\mathbf{J} . \mathbf{K}^{-1} \cdot \mathbf{m o l}^{-1}\end{array}$} & $\begin{array}{c}\mathbf{S}^{\mathbf{o}} \\
\mathbf{J} \cdot \mathbf{K}^{-1} \cdot \mathbf{m o l}^{-1}\end{array}$ \\
\hline Keto form & -4747.54 & \multicolumn{2}{|l|}{81.66} & \multicolumn{2}{|c|}{2951.87} & \multicolumn{2}{|c|}{-4746.35} & -4746.51 & 935.41 & & 1454.22 \\
\hline Enol form & -4746.77 & \multicolumn{2}{|l|}{81.80} & & 2888.82 & \multicolumn{2}{|c|}{-4745.60} & -4745.77 & 922.18 & \multicolumn{2}{|r|}{1429.77} \\
\hline \multicolumn{12}{|c|}{ DOS calculations } \\
\hline & $\begin{array}{c}\text { E }_{\text {LUMO }} \\
/ \mathrm{eV}\end{array}$ & $\begin{array}{c}\mathbf{E}_{\text {номо }} \\
/ \mathrm{eV}\end{array}$ & $\mathbf{E}_{\mathrm{g}} /$ & & $\eta / e V$ & $\mu / \mathrm{eV}$ & $\sigma / \mathrm{eV}$ & $\omega / \mathrm{eV}$ & S/eV & $\begin{array}{c}\Delta \mathbf{E}_{\mathrm{b}-\mathrm{d}} \\
/ \mathbf{e V}\end{array}$ & $\Delta \mathbf{N}$ \\
\hline Keto form & 5.23 & 3.15 & 2.0 & & -4.19 & 1.04 & -0.04 & 8.44 & 0.48 & -0.26 & 3.69 \\
\hline Enol form & 5.80 & 3.16 & 2.6 & & -4.48 & 1.32 & -0.32 & 7.60 & 0.38 & $\begin{array}{l}-0.33 \\
\end{array}$ & 6.16 \\
\hline
\end{tabular}

By studying the electrical structure, the conductivity and transfer of electrons in the anvil form is up to about more than keto, but in general, the introduction of folic acid into the complex structure causes the structural balance of the drug and improves the transfer and release of the drug to the target cell. Therefore, folic acid increases the solubility, increases the drug ability and increases the biocompatibility of nanographene oxide.

Table 4 shows the Ead adsorption energy for the keto and enol forms of the direct drug complex with nano-graphene oxide and with the folic acid/nano-graphene oxide intermediate calculated by Eq.(1), that indicates a greater tendency of the drug to be absorbed by this complex in the form of ketoes, which confirms the stability of the structure and the greater the likelihood of this complex in aqueous medium.

Table 4. Calculated adsorption energy for keto and enol forms of NGO/CA and NGO/FA/CA complexes.

\begin{tabular}{|c|c|c|}
\hline $\begin{array}{c}\text { Ead } \\
\text { a.u. }\end{array}$ & NGO/CA & NGO/FA/CA \\
\hline Keto form & 143.45 & 65.24 \\
\hline Enol form & 144.22 & 77.62 \\
\hline
\end{tabular}

\section{- IR spectroscopy}

The camptothecin is a polycyclic alkaloid with two forms of lactone and carboxylate. Figure 5 shows the IR spectrum of the drug structure. The tensile vibration of carboxylate form is in the range of $1349-1289 \mathrm{~cm}^{-1}$ for CO-bonding. The presence of spectra in the range of $2300-2050 \mathrm{~cm}^{-1}$ belongs to the tensile vibrations $-\mathrm{C}=\mathrm{N}$ - and the absorption peak observed in $3772 \mathrm{~cm}^{-1}$ belongs to the tensile vibration $\mathrm{O}-\mathrm{H}$. 
One of the principles of drug transfer is to maintain its structure by drug carriers, in other words, by creating a covalent and non-covalent interaction, the structure of the drug should not change and by maintaining the structure, it can be delivered to the target cell. Figure 5, parts (a) and (b) of the IR spectrum of the drug complex/nanographene oxide is an unmediated compound, with (a) in Fig.5 is the anol form and (b) is the keto form of camptothecin in the nano-composite. Or displaced, indicating the instability of the drug structure in direct binding to nano-graphene oxide.

In $c$ and d parts of Figure 5, the IR spectrum shows the structure of the drug binding to the intermediate of folic acid, the (c) part shows the enol form and (d) is the keto form. Increased absorption intensity of $\mathrm{C}-\mathrm{O}$ group confirms the binding of the drug to the complex and indicates the stability of the drug structure in the NGO/FA/CA complex.

In addition, due to the presence of folic acid, it transfers the drug to the target cell and provides the necessary $\mathrm{pH}$ for the lactony/carboxylate balance to the ketone form. Also, the presence of nano-graphene oxide has made it more soluble in aqueous medium deposition in the lumens and removal of hemorrhagic cystitis after taking the drug. The NGO/FA/CA complex, in addition to solving the problem of drug solubility and its instability at physiological $\mathrm{pH}$, is potentially able to target, reduce drug resistance, and reduce clearance and so on.
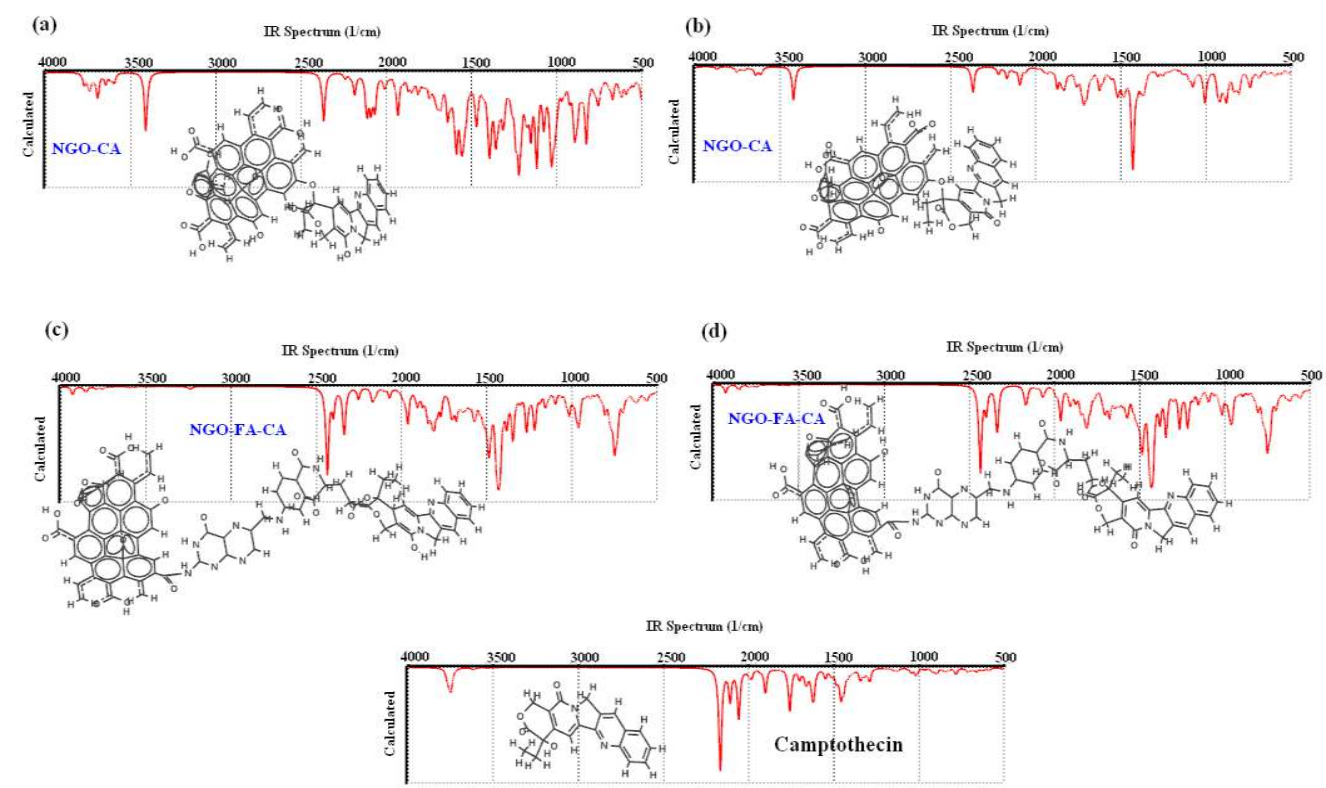

Figure 5. Computational IR spectrum a) enol form and b) camptothecin/nano-graphene oxide complex ketones and c) enol form and d) camptothecin/folic acid/nano-graphene oxide complex ketones.

In general, the interaction of the camptothecin anticancer drug in complex with nanographene, nano-graphene oxide and nano-graphene oxide modified with folic acid, 
shows that the maintenance of the drug structure using folic acid intermediate despite covalent bonding is better than other cases. In experimental nanomaterial activities, when anticancer drugs are formulated and used as drug nano-systems, they show more toxic effects on resistant cells than free drug, which can be achieved by releasing and releasing the drug using Nanoparticles and acidic intermediates, make the drug more effective. This indicates that nanoparticles are able to bypass the cell surface transporter pathway due to the entry of these nanoparticles through specific or nonspecific endocytosis pathways into the cell $[28,29]$.

\section{Conclusion}

The presence of nano-graphene oxide nanoparticles due to the functional groups on its edge increases the solubility in aqueous medium equivalent to $\left(8.3 \mathrm{mg} \cdot \mathrm{ml}^{-1}\right)$ of the drug and to stabilize the lactone ring and increase the effectiveness of folic acid as an intermediate in this study has been used. According to studies on increasing the effectiveness of the drug, nano-graphene oxide has advantages over nano-graphene, such as having hydrophilic functional groups. Brief Toxicity of nano-graphene oxide can be reduced by modification by polymers and other nanoparticles.

The calculations based on M06-2X/6-31G ** are investigated for the binding of $\pi-\pi$ of camptothecin to the nano-graphene surface, covalent bonding with nano-graphene oxide and folic acid-modified nano-graphene oxide in aqueous medium. According to the calculated and structural data, the drug has maintained its structure by binding to the folic acid/nano-graphene oxide complex in aqueous medium and is predominant its lactone form. As can be seen in Table 3, the energy gap is calculated for the enol form, it is greater for this structure, which indicates the softening of the structure. While the lactone ring shows a more rigid structure in this complex. The adsorption energy $\left(E_{a d}\right)$ is calculated for the enole and keto forms of both bonds, is indicates that the ketone form in the NGO/FA/CA complex is 65.24 a.u., It shows a greater tendency of the drug to interact with this nano-surface, which reduces the side effects of the drug. Therefore, graphene oxide/folic acid can be considered as a drug transporter with fewer and weaker side effects. These drugs can help doctors perform a multilateral attack on tumors and kill cells before they become more resistant to the compounds. Because cancer cells are rapidly resistant to drugs and also drugs for various cancers do not respond to other types of the disease, it is better to fight these tumors with several drugs at once to make these cancer cells resistant. Drug 
compounds become harder. Nanoparticles and drug are linked by a chemical bond. While the drug is stored in other nanoparticles used for transport by polymer networks or in a fat capsule, the drugs inside these new nanoparticles are held together by a chemical bond. These drugs are released during the breakdown of a chemical bond, the process of which takes place to some extent under the control of an unknown enzyme in the body. Systematic nano-graphene oxide can be shown that nanographene oxide hybrids with other biocompatible polymers can show better results when combined with tumor-specific antibodies for targeted delivery.

\section{Declarations}

Funding: There is no information that explains whether and by whom the research was supported.

Availability of data and material: All data and calculations are transferable and observable.

Code availability: Wave function Spartan 14 v1.1.4

\section{Authors' contributions:}

Both authors have participated in: conception and design, acquisition of data, analysis and Interpretation of data, drafting of the manuscript, critical revision of the manuscript for important intellectual content and so on.

\section{References}

[1] F.Din, W.Aman, I.Ullah, O.S.Qureshi, O. Mustapha, S. Shafique, A. Zeb. Effective use of nanocarriers as drug delivery systems for the treatment of selected tumors. Int $\mathbf{J}$ Nanomedicine. 12(2017)7291-7309.

[2] S. Senapati, A. K. Mahanta, S.Kumar, P. Maiti. Controlled drug delivery vehicles for cancer treatment and their performance. Signal Transduct Target Ther. 3:7(2018)1-19.

[3] J. K.Patra, G. Das, L. F. Fraceto, E. V. R. Campos, M. P. Rodriguez-Torres, L. S. Acosta-Torres, L. A. Diaz-Torres, R.Grillo, M. K. Swamy, S. Sharma, Solomon Habtemariam, and H.S. Shin. Nano based drug delivery systems: recent developments and future prospects. J Nanobiotechnology. 16:71(2018).

[4] J.Liu, L.Cui, D.Losic. Graphene and graphene oxide as new nanocarriers for drug delivery applications. Acta Biomaterialia. 9:12 (2013) 9243-9257.

[5] S.A. Özkan, A. Dedeoĝlu, N. K. Bakirhan, Y. Özkan. Nanocarriers Used Most in Drug Delivery and Drug Release: Nanohydrogel, Chitosan, Graphene, and Solid Lipid. Turk J Pharm Sci. 16:4 (2019) 481-492.

[6] C.M.Callion, J.Burthem, K. Rees-Unwin, A.Golovanov, A.Pluen. Graphene in therapeutics delivery: Problems, solutions and future opportunities. European Journal of Pharmaceutics and Biopharmaceutics. 104 (2016) 235-250. 
[7] S. Syama, P. V. Mohanan. Comprehensive Application of Graphene: Emphasis on Biomedical Concerns. Nano-Micro Lett. 11:6 (2019) 1-31.

[8] J. Feng, Y.Ye, M. Xiao, G. Wu, Y. Ke. Synthetic routes of the reduced graphene oxide. Chemical Papers. 74 (2020) 3767-3783.

[9] M.E. Wall, M.C. Wani, C.E. Cook, K.H. Palmer, A.T. McPhail, G.A. Sim. Plant Antitumor Agents. I. The Isolation and Structure of Camptothecin, a Novel Alkaloidal Leukemia and Tumor Inhibitor from Camptotheca acuminate. J. Am. Chem. Soc. 88:16 (1966) 3888-3890.

[10] Wavefunction Spartan 14 v1.1.4.

[11] H. Liu, J.Y. Lee, Electric Field Effects on the Adsorption of CO on a Graphene Nanodot and the Healing Mechanism of a Vacancy in a Graphene Nanodot, J. Phys. Chem. C. 116 (2012) 30343041 .

[12] M. Zaboli, H. Raissi, N. Rahmani Moghaddam, F. Farzad. Probing the adsorption and release mechanisms of cytarabine anticancer drug on/from dopamine functionalized graphene oxide as a highly efficient drug delivery system, Journal of Molecular Liquids. 301 (2020) 112458.

[13] Y. Zhao, D.G. Truhlar, Density functionals with broad applicability in chemistry. Acc. Chem. Res. 41 (2008) 157-167.

[14] G. A. Petersson, M. J. Frisch, F. Dobek, B. Zulueta.Three-Body Dispersion Corrections to the Spherical Atom Model: The PFD-3B Density Functional. J. Phys. Chem. A. 124(49) (2020) 10296-10311.

[15] L. Mahdavian, DFT studies of the drug carrier of anti-migraine (sumatriptan) on nano-graphene oxide (NGO) and graphene oxide/polyethylene glycol polymer nano-composite, Diamond and Related Materials. 104 (2020) 107745.

[16] M.E. Belghiti, S. Echihi, A. Mahsoune, Y. Karzazi, A. Aboulmouhajir, A. Dafali, I. Bahadur. Piperine derivatives as green corrosion inhibitors on iron surface; DFT, Monte Carlo dynamics study and complexation modes. Journal of Molecular Liquids. 261(2018) 62-75.

[17] M. Shahabi, H. Raissi. Investigation of the solvent effect, molecular structure, electronic properties and adsorption mechanism of Tegafur anticancer drug on Graphene nanosheet surface as drug delivery system by molecular dynamics simulation and density functional approach. J. Incl. Phenom. Macrocycl.Chem. 88:3-4 (2017) 159-169.

[18] K. Rohini, D.M. Sylvinson R.S. Swathi,. Intercalation of HF, H2O, and NH3 Clusters within the Bilayers of Graphene and Graphene Oxide: Predictions from Coronene-Based Model Systems. J. Phys. Chem. A. 119 (2015) 10935-10945.

[19] J. Liu, L. Cui, D. Losic. Graphene and graphene oxide as new nano-carriers for drug delivery applications. Acta Biomaterialia. 9:12 (2013) 9243-9257.

[20] S. S. Banerjee, N. Aher, R. Patil, J. Khandare.Poly (ethylene glycol) -Prodrug Conjugates: Concept, Design, and Applications. Journal of Drug Delivery. 2012:103973 (2012) 1-17.

[21] Z. Hasanzade, H. Raissi. Solvent/co-solvent effects on the electronic properties and adsorption mechanism of anticancer drug Thioguanine on Graphene oxide surface as a nanocarrier: Density functional theory investigation and a molecular dynamics. Applied Surface Science. 422 (2017) 1030-1041.

[22] A. D. Putri, B. T. Murti, S. Kanchi, M. I. Sabela, K. Bisetty, A. Tiwari, I .M. Asiri, A. M. Asiri. Computational studies on the molecular insights of aptamer induced poly $(\mathrm{N}$ isopropylacrylamide)-graft-graphene oxide for on/off- switchable whole-cell cancer diagnostics. Scientific RepoRts 9:7873 (2019) 1-14. 
[23] D. Veclani, M. Tolazzi, A. Melchior. Molecular Interpretation of Pharmaceuticals'Adsorption on Carbon Nanomaterials: Theory Meets Experiments. Processes, 8:642 (2020)1-39.

[24] D. W. Boukhvalov , M. I. Katsnelson. Modeling of Graphite Oxide. J. Am. Chem. Soc. 130(32) (2008) 10697-10701.

[25] M. Pumera. Electrochemistry of graphene: new horizons for sensing and energy storage. Chem Rec. 9(4) (2009) 211-23.

[26] A.Tas, N. K. Cakmak, Synthesis of PEGylated nanographene oxide as a nanocarrier for docetaxel drugs and anticancer activity on prostate cancer cell lines. Human \& Experimental Toxicology. 40(1) (2021) 172-182.

[27] Z. Zhang, R. Yang. Novel nanocomposites based on hydroxyethyl cellulose and graphene oxide. Fibers and Polymers. 18 (2017) 334-341.

[28] O. P. Troncoso, F. G. Torres. Bacterial Cellulose-Graphene Based Nanocomposites. Int. J. Mol. Sci, 21(17) (2020) 6532 (1-17).

[29] H. Sharma, S. Mondal. Functionalized Graphene Oxide for Chemotherapeutic Drug Delivery and Cancer Treatment: A Promising Material in Nanomedicine. Int. J. Mol. Sci, 21(17) (2020) 6280 $(1-17)$. 

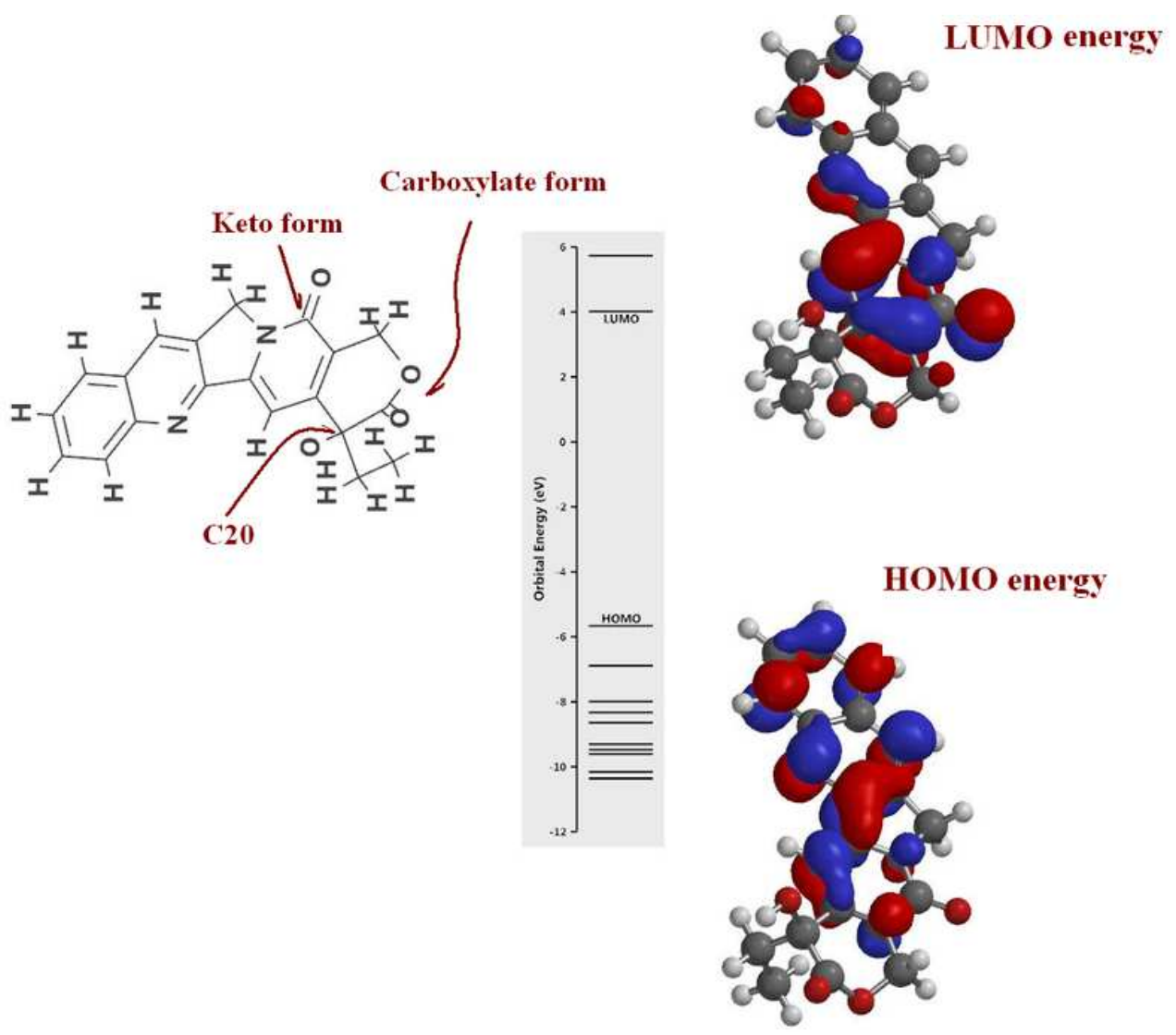

\section{Electrostatic potential map}

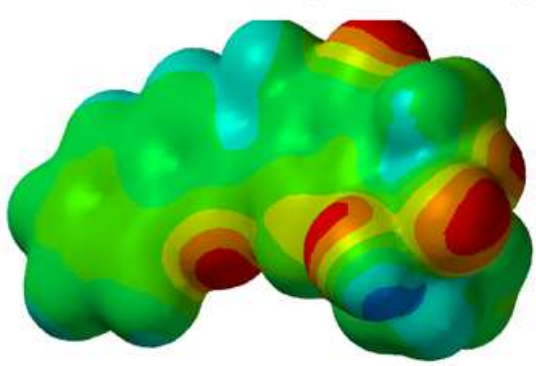

\section{Figure 1}

Geometric and optimized structure of the anticancer drug of camptothecin and its lactone and carboxylate forms.

(a)

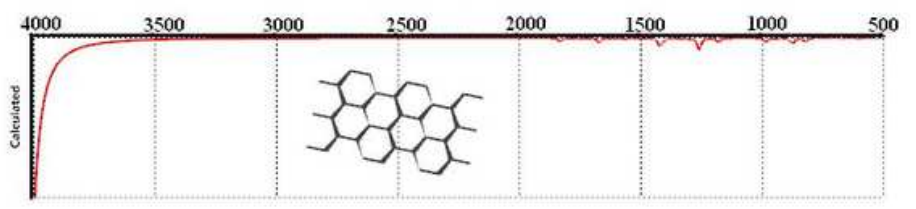

(b)

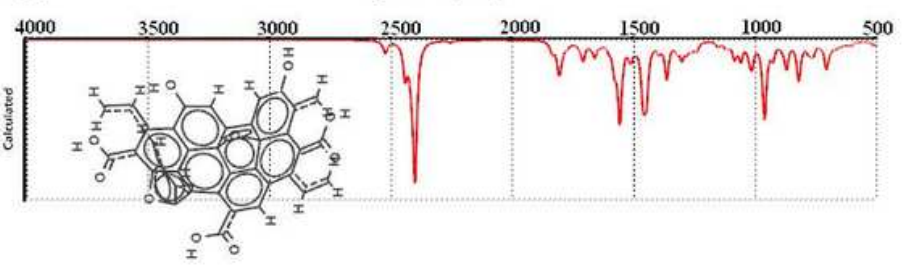

(c)

IR Spectrum $(1 / \mathrm{cm})$

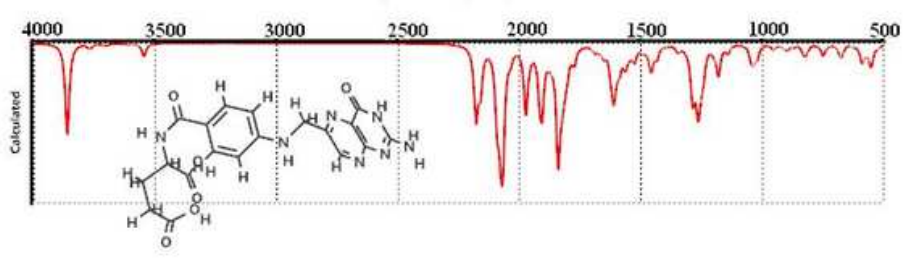

Figure 2

IR spectrum with optimized structure a) graphene, b) graphene oxide and c) folic acid. 


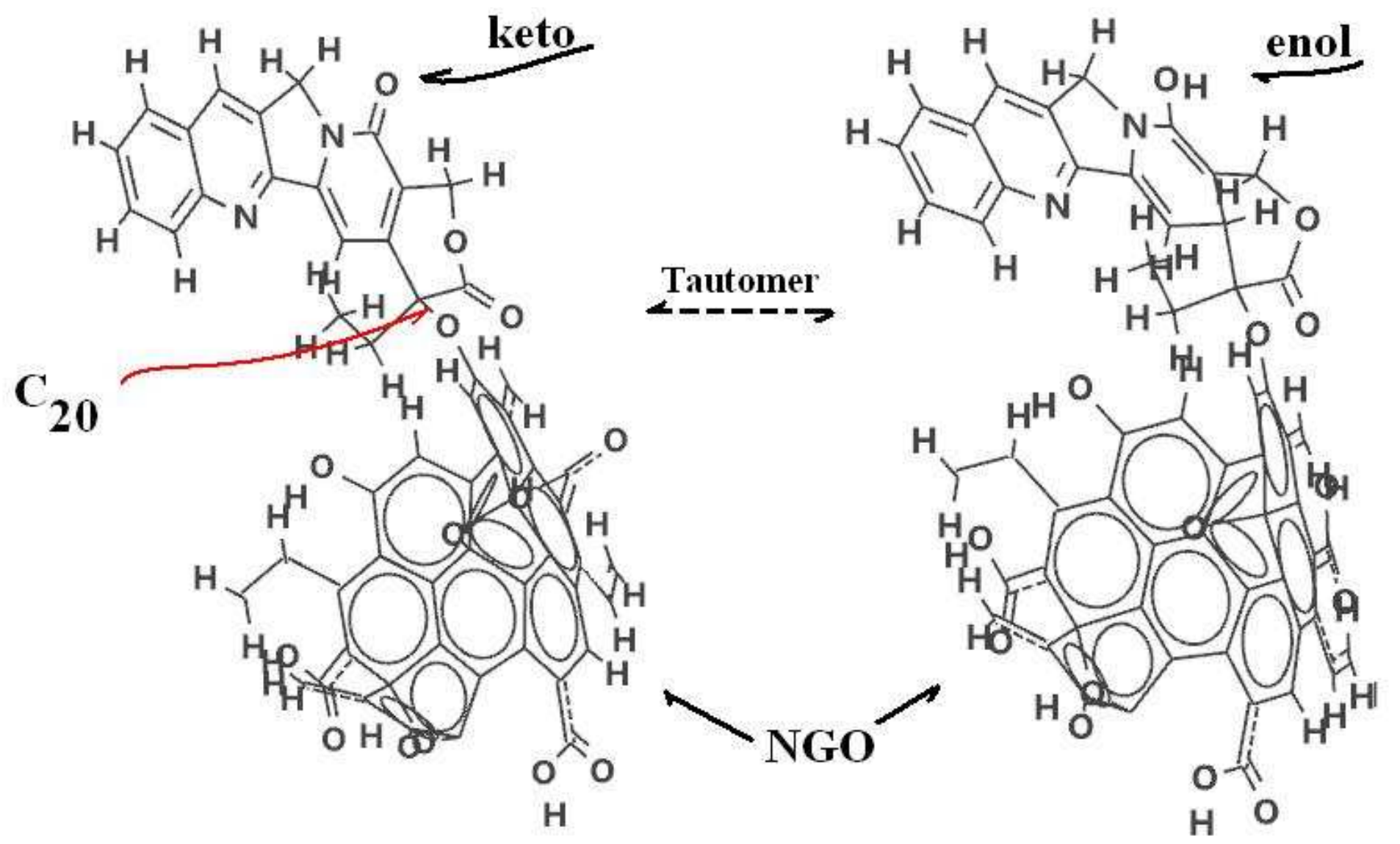

Figure 3

Optimized structure of binding of the anticancer drug of camptothecin to nano-graphene oxide as ketone form totomerism. 


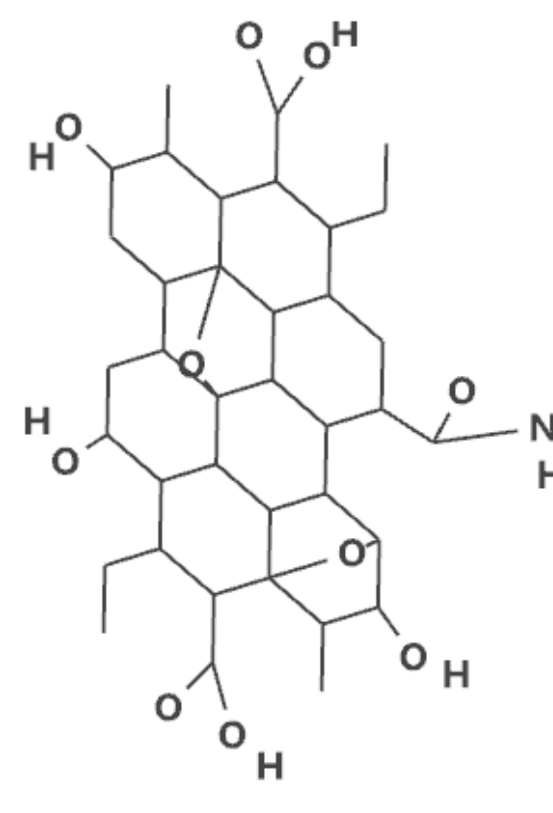

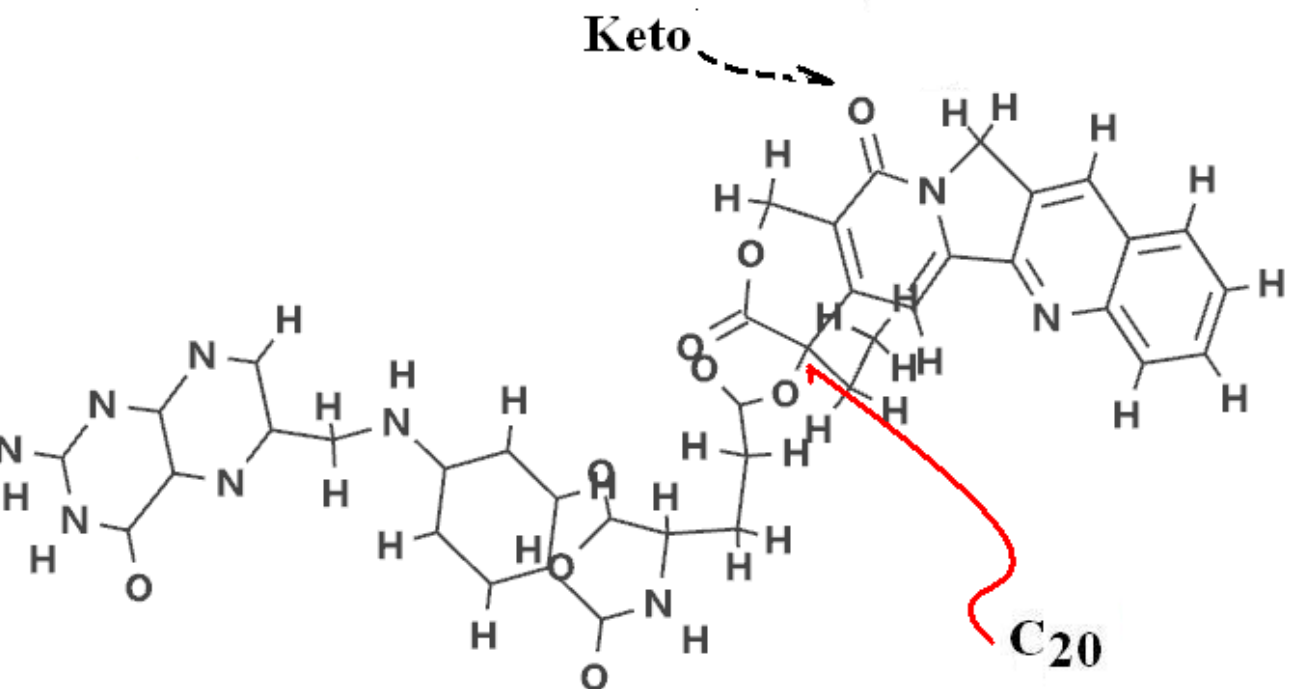

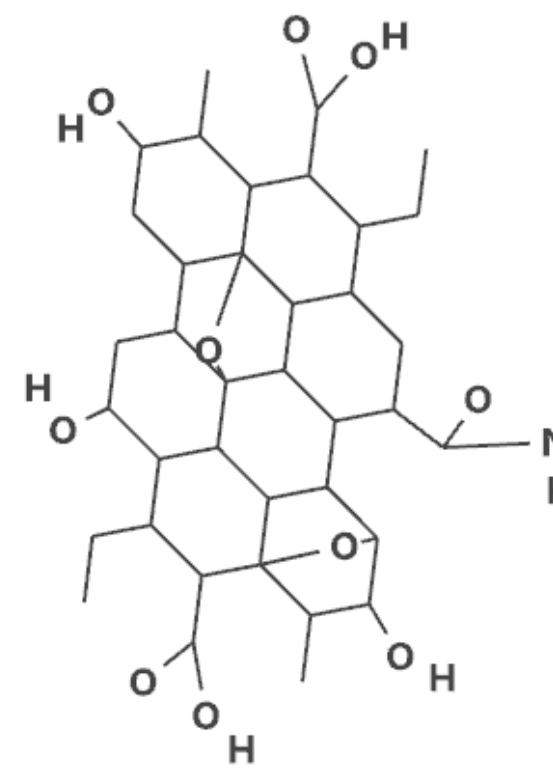<smiles></smiles>

Figure 4

Optimized structure of the camptothecin anticancer drug with folic acid/nano-graphene oxide nanocomposite in totomerism of enol『ketol. 
(a)

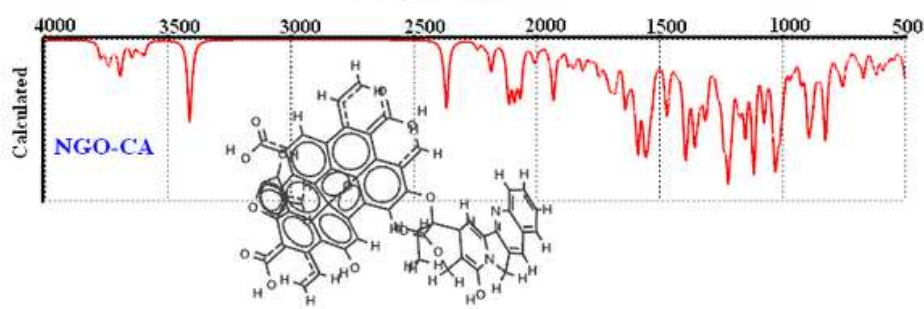

(c)

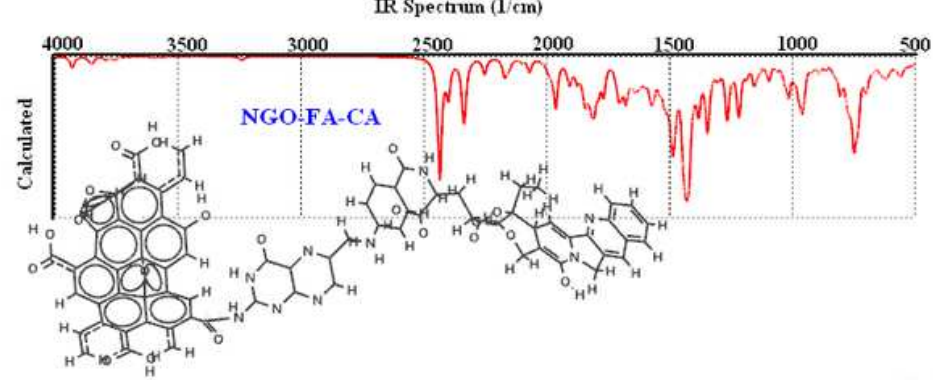

(b)

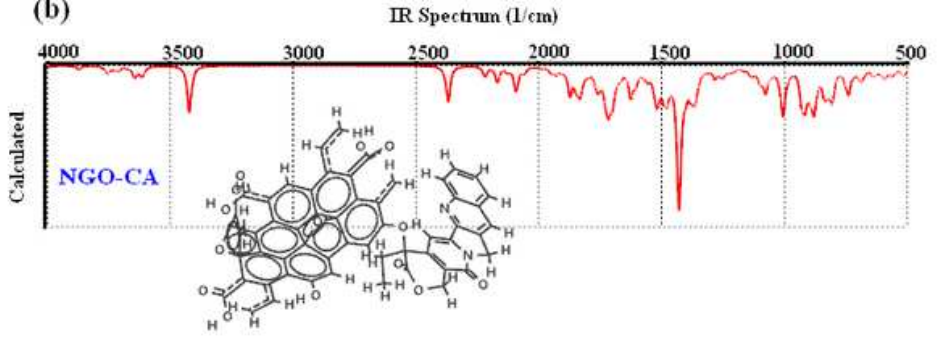

(d)

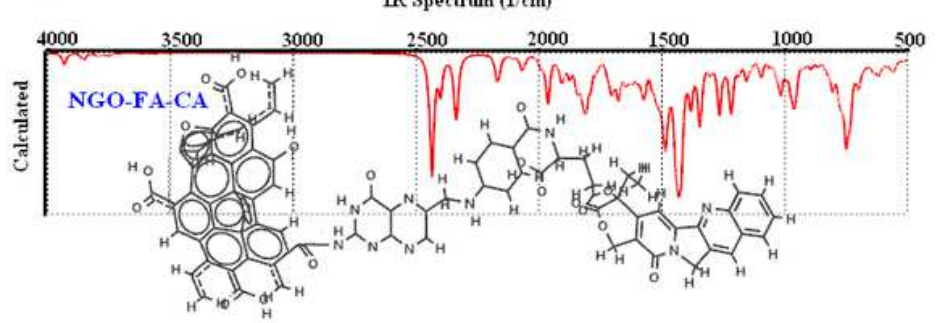

IR Spectumum $(1 / \mathrm{cm})$

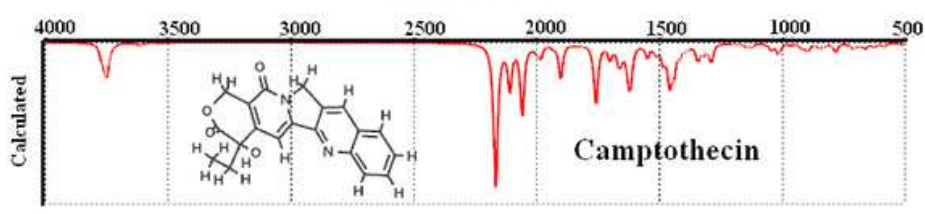

\section{Figure 5}

Computational IR spectrum a) enol form and b) camptothecin/nano-graphene oxide complex ketones and c) enol form and d) camptothecin/folic acid/nano-graphene oxide complex ketones. 